\title{
Photonic power delivery through optical fiber using very high power laser diode arrays
}

\author{
Matthew Heino, Robert Saethre \\ Bechtel Nevada \\ 5667 Gibraltar Dr., Pleasanton, CA. 94588
}

\begin{abstract}
Described is a system that will provide isolated electric power for a circuit that drives the core reset of a pulsed power modulator. This can be accomplished by coupling light from a number of diode laser bars to bundles of $200 \mu \mathrm{m}$ multimode optical fibers. This is then coupled to photo-voltaic power converters that will deliver $16 \mathrm{~V} 29 \mathrm{~mA}$ of electricity from 1 watt of optical power. Spot size at the bundle face is a Gausian ellipse with a major axis of $1.4 \mathrm{~mm}$ radius and a minor axis of $0.118 \mathrm{~mm}$ with a maximum full angle divergence of $16 \mathrm{X} 2.4$ degrees. Data is presented from four $20 \mathrm{~W}$ laser bars coupled to four bundles of 12 tibers generating a total of $24 \mathrm{~W}$ of electrical power. Various schemes are used to maximize coupling into the optical fiber while limiting the number of optical components, and comparing components such as fresnel and aspheric lenses and lens ducts for effectiveness and cost. This will provide a completely isolated low power source for high voltage, high current environments where traditional isolation techniques yield inadequate isolation or prove too cumbersome.
\end{abstract}

Keywords: fiber-optics, solid-state lasers, photo-voltaic power, Pulsed power systems 


\section{Introduction}

In many high power systems, particularly those using power MOSFETs switch high currents and voltage from a low power signal. The risk here is when these power MOSFETs are stacked and paralleled, the currents and voltages become very high indeed, these high electric and magnetic fields can easily generate unwanted signals in the driver circuitry, and damage components from inducted currents in the lines that power the driver. The present drive towards compact, high energy density packaging requires another way to obtain high isolation. One solution is to generate the necessary low power right at the board using photovoltaic cells illuminated by laser light transmitted by fiber optics.

The need that initiated this project originated with the Advanced Radiographic Machine (ARM) an all solid state pulsed power modulator ${ }^{7}$ for use in a linear induction accelerator. This machine uses stacks of FETs connected in parallel and capacitors in series about a metglass core with pulse switching and core reset switching stacks next to each other. These rings then can be connected together to form a voltage adder. The next version of this machine with have the reset stacks in an inner ring, and the switching stacks forming an outer ring, thus enhancing the compactness and power of this type of modulator. There exists, however, a high voltage plane between the reset stack and the outside world. Add to that there is high voltage between the boards themselves, which is of the main concern. The only way to pass power and signals to this stack is through fiber-optic connections or highly shielded conventional power isolators. This paper describes an investigation of power transmission using only light

\section{Laser source}

With a growing market for high average power laser diode bars, their cost has become quite reasonable ${ }^{5}$. Just a few years ago this type of photonic power system would have been prohibitively expensive. We purchased unmounted $20 \mathrm{~W} \mathrm{cw}$ laser bars with resonator length of $1,000 \mu \mathrm{m}$ array length of $1 \mathrm{~cm}$ and height of $140 \mu \mathrm{m}$. These lasers have a full angle divergence of 10 by 38 degrees and output at $808 \mathrm{~nm}$. The P-side was soldered to a diamond heatspreader, which in turn was soldered to a copper submount heatsink using an indium/silver/lead solder. The $\mathrm{N}$-side was soldered to a strip of copper foil. Active cooling was employed using thermal electric coolers of high $\wedge \mathrm{Q}$, about $22 \mathrm{~W}$ per bar. Precise wavelength was not required for this application, so temperature control was omitted. The lasers output $20 \mathrm{~W}$ optical power at a nominal drive current of 23 amps at 65 degrees $F$.

Four of these lasers were connected in series to a DC power supply and simple driver circuit. This provided 48 channels. However, it is possible to drive up to two sets of four laser bars in parallel generating $160 \mathrm{~W}$ of optical power along 96 channels. The whole system is then enclosed to a Class I embedded status with only optic fibers exiting the enclosure. This system of laser, chiller, and fiber coupling is packaged in a box with dimensions of only $30 \mathrm{~cm}$ by $45 \mathrm{~cm}$ by $16 \mathrm{~cm}$ !

\section{Coupling to Fiber}

The challenge we were presented with was not just coupling a laser to fiber. We needed a system that was effective, but also as inexpensive as possible. We also needed a specific power per fiber optic channel, 1 watt. Therefore the number of optical elements had to be minimized, and novel materials used. For example, to condition the very divergent fast axis we used a section of all core $600 \mu \mathrm{m}$ fiber optic as a cylindrical lens $\mathrm{s}^{2,4}$. This reduced the divergence of the fast axis from $38^{\circ}$ to $2^{\circ}$. We decided to investigate the effectiveness and cost of two optical choices, using commercial grade aspheres and cylindrical lenses to collect light to a fiber bundle sized spot, and to try a lens duct. Zemax ${ }^{\text {tm }}$ code was used to model the beam shaping of these two choices. These models lead us to believe the lens duct to be the ideal choice due to superior beam quality, and its fantastically low cost. Also using the lens duct ${ }^{4}$ means the only other optical elements needed is a $600 \mu \mathrm{m}$ 
fiber for diode conditioning ${ }^{4}$, and a $1,000 \mu \mathrm{m}$ fiber for final coupling to the bundle ${ }^{2}$, which satisfies the requirement for minimal cost.

\section{Asphere's and cylinders.}

With the laser fast axis conditioned with a fiber lens, we used an odd asphere as a condenser lens and a commercial grade cylinder lens to condition the slow axis. This has the advantage of condensing the light in a diffraction limited manner and the aspherical shape is less susceptible to the fact that the laser source is indeed not a single source but a linear array of emitters, and so creates a smooth Gaussian ellipse at the waist. The resultant spot is now the same size and the line bundle of fiber I'm coupling to. I now use a $1 \mathrm{~mm}$ fiber lens to couple this spot into the bundle. The resultant entrance angles are now

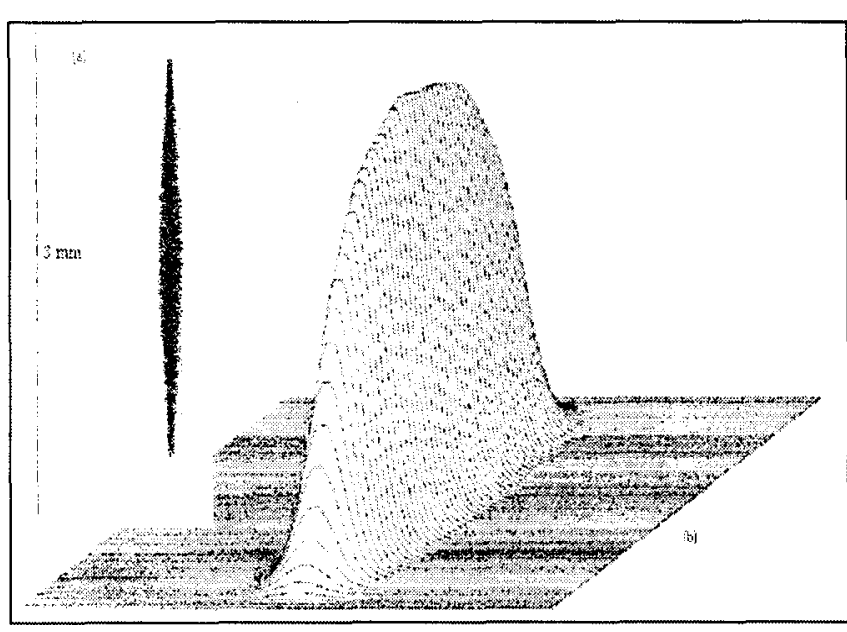

Figure 1. (a)Modeled Spot size at the entrance face of the fiber bundle (b) Modeled intensity profile at same location
0.34 in the fast axis and 0.04 in the slow axis (notethe label fast and slow axis are maintained in reference to the laser, even though at points in the optical train the 'slow' axis is the 'faster' axis). Figure (1) shows the model and actual intensity profiles as seen at the bundle face. Due to the very high power density at the bundle face, we employ a copper scraper to prevent diffracted or abberated parts of the beam (or misalignments) from burning the epoxy that holds the bundle. While this scheme is adequate, we feel it is not the best choice. There are four optical elements, two of which can be fairly expensive. Even assuming 98\% transmission for each element, this means we lose $8 \%$ of the total light passing through this train, or about 2 watts of power which cost as much as $\$ 40$ to produce. Further we now have four elements which must have very tight alignment tolerances for maximum coupling, which is difficult to achieve with out precise machining which also is expensive. We feel that for this application, conventional optical elements are not the answer.

\section{Lens Duct with fiber lens'}

The laser output is still conditioned with a $600 \mu \mathrm{m}$ fiber lens. Immediately following is rectangular segment of glass with surface one being polished to a sphere of radius $23 \mathrm{~mm}$ and surface two being plano. From surface one to two the glass is tapered from a $15 \mathrm{~mm}$ by $2 \mathrm{~mm}$ cross section to a $3 \mathrm{~mm}$ by $2 \mathrm{~mm}$ cross section at the plano surface. Figure 2 shows the intensity profile in the model ,which shows good agreement with the results of R.Fu et al ${ }^{\prime}$, and the actual profile. At $3 \mathrm{~mm}$ the spot shows as a Gausian ellipse with the major axis the size of the the fiber bundle line. Again a $1 \mathrm{~mm}$ fiber lens is used to make a $2.8 \mathrm{~mm}$ by 0.250

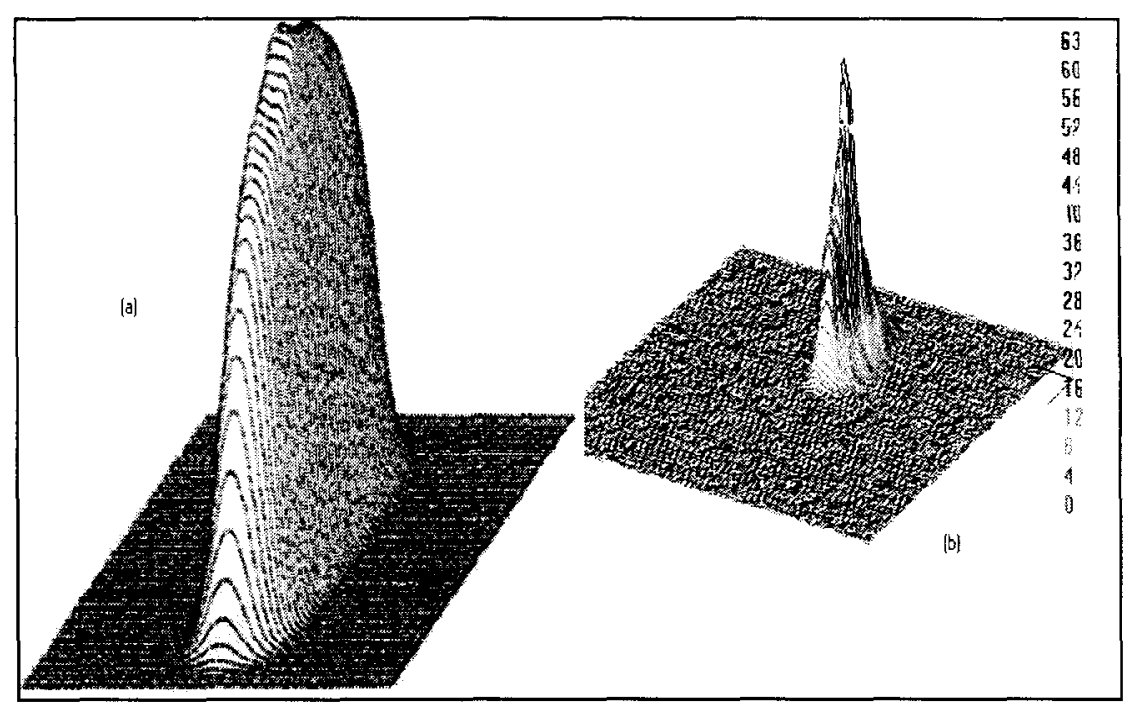

Figure 2 (a) Simulated profile at $3 \mathrm{~mm}$ from the exit of the duct.(b) actual beam exiting the duct at the same location. Note: scale is not preserved. 
$\mathrm{mm}$ line with entrance angles of 0.08 in the fast axis and 0.18 in the slow axis

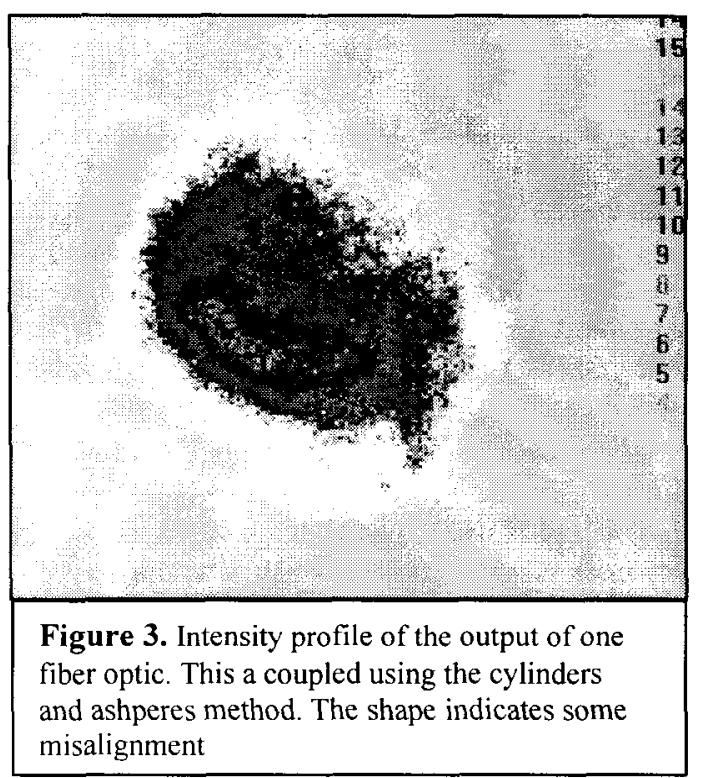

which is well within the NA of the optical fiber transmission lines.

Only three elements were used in this train, and each component has very low cost. In fact the tested lens duct was made from a typical glass slide that was cut and polished to shape. Alignment is also simplified by using a ceramic sub-mount with v-grooves that will hold laser, heat-spreader and optics all in one plane.

\section{Fiber Bundle}

Each bundle consists of a linear array of $12200 / 230 \mu$ m multi-mode fibers in a close pack. Figure 3 shows the output profile detail of one channel. Figure 4 shows the profile of all 12 , we can see each fiber carries nearly the same power, so each board sees a similar power feeding the storage capacitors. With the bundle face being slightly over filled and evanescent coupling due to fiber proximity in the bundle, it is expected each channel to have the same or nearly the same power level.

\section{CONCLUSIONS}

The photocells we used were purchased from a vendor, so their construction will not be discussed here. They are rated to produce $16 \mathrm{~V}$ in open circuit and $30 \mathrm{~mA}$ in short circuit when illuminated with 1 Watt of optical power ${ }^{8,9}$ in the range of 800 to $850 \mathrm{~nm}$. We will use this mark as an indicator of adequate power coupled per fiber. It should be kept in mind that in this package the power density on the photocell at one watt is nearing saturation and the damage threshold ${ }^{9}$. This is due to the fact that size was mandated to be small. If power needs out weigh size constraints, receiver packages could be made to suit quite readily.

It is required to have uniformity of illumination on the photo receiver to prevent portions of the photocell to be reverse bias and become resistors. With this in mind we see that the formation of cladding modes must be avoided.. As tested this system is not suited for long term 'on' operation. This was not a requirement for the project it supports, boards would be powered up, the machine fired, and the laser power turned off. The power density at the bundle face is about $1 \mathrm{kw} / \mathrm{cm}^{2}$. Most epoxy will burn at this level. To minimize this we installed a mirror scraper around the fiber-lens. Coupling efficiency for both methods was less than predicted, with the lens duct yielding better results. I suspect skew alignment as a major cause. Also there is evidence of outcoupling of light into the epoxy that bound the bundle. This could be solved using an opaque epoxy, or to construct a bundle using silicon v-groove sub-mounts.

Total electrical efficiency is rather low, but it is a good system where the gains in the end product are worth the cost, which in large numbers can be as low as $\$ 20$ per channel. Future designs are in progress that will reduce cost per channel, and

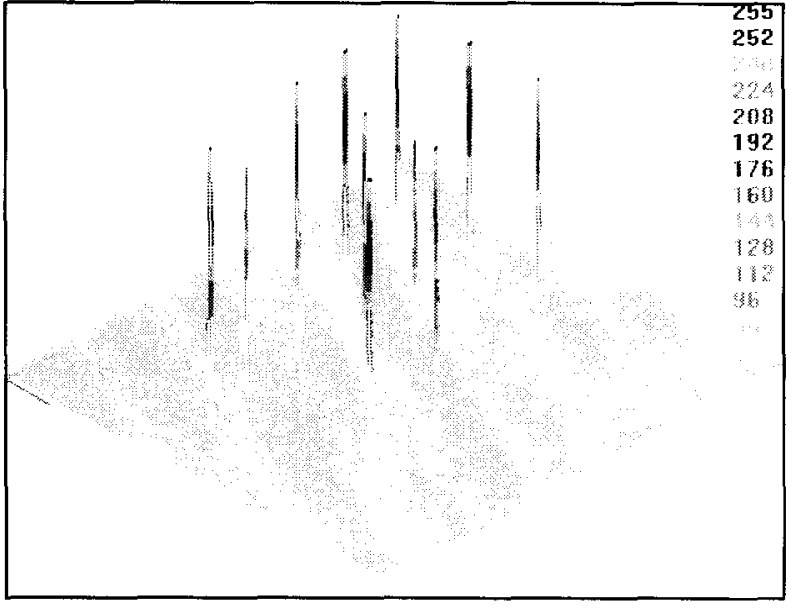

Figure 4. 12 fiberes were inserted on a grid and imaged onto a Cohu camera stopped to f1 1 and attenuated with a ND2.5 filter. Coupling achieved with aspheres and cylinders method increase overall electrical efficiency, as high as $37 \%$ !

\section{ACKNOWLEGEMENTS}

This work was supported by Department of Energy contract \# DE-AC-08-96NV11718 and performed at I awrence L ivermore National Laboratory.

I would also like to acknowledge the generous support from: D.Mitchell, S.Meyers,P.Watts, C.Brooksby (Bechtel Nevada), H.Kirbie, R.Hanks, B.Lee, B. Hickmann (LLNL). 
1. R.Fu,G.Wang,Z.Wang,E.Ba,G.mu and X.Hu, "Design of efficient lens ducts", App.Optics Vol.1.no.18 p4000-4003, June 1998.

2. R.Wilson, "Ball-lens coupling efficency for laser diode to singlemode fiber: comparison of independent studies by distinct methods", App. Optics Vol.37,no.15 pp 3201-3205,May 1998.

3. S.Mondal, S.Gangopadhyay, and S.Sarkar, “Analysis of an upside-down taper lense end from a single-mode step-index fiber", App.Optics Vol.37, no.6, pp 1006-1009, Feb 1998.

4. R.Beach,M.Emanuel,B.Freitas,J.Skidmore,N.Carlson,W.Benett, and R.Solarz, "Applications of microlens-conditioned Laser Diode Arrays", SPIE Vol.2383 pp.283-297, Feb 1997.

5. M.Emanuel,J.Skidmore, and R.Beach, "High-Power Laser Diodes at Various Wavelengths", SPIE Vol.3001 pp.2-6, Feb.1997.

6. R.Beach,M.Emanuel,W.Benett,B.Freitas,D.Ciarlo,N.Carlson,S.Sutton,J.Skidmore, and R.Solarz, "Improved performance of high average power semiconductor arrays for applications in diode pumped solid state lasers" ,SPIE Vol.2148, pp.13-29. Jan. 1994.

7. H.Kirbie,B.Hickman,B.Lee,C.Ollis,C.Brooksby, and R.Saethre, "An all solid state pulse power source for high PRF induction accelerators", Proceedings of the 23rd International Power Modulator Symposium, Rancho Mirage, CA, June 22-25, 1998, UCRL-JC-130691. To be published.

8. G. Landis, P. Jenkins, R. Lowe and R. Jain, "Solar Cells for Laser Power Receivers," Tech. Digest 7th International Photovoltaic Science \& Engineering Conf., Nov. 1993, Nagoya Japan, 616.

9. G. Landis, "Photovoltaic Receivers for Laser Beamed Power," Journal of Propulsion and Power, Vol. 9 No. 1, 105-112 (1993). Presented at 22nd IEEE Photovoltaic Specialists Conference, Las Vegas NV, Oct. 1991, Vol. II, 1494-1502. Also available as NASA Report CR-189075 (1991).

10 G. Landis, "Applications for Space Power by Laser Transmission," SPIE Optics, Electro-optics \& Laser Conference, Los Angeles CA, Jan. 24-28, 1994; Laser Power Beaming, SPIE Proceedings Vol. 2121, 252-255. 